\title{
A Numerical Study on the Dimension of an Extremely Inhomogeneous Matter Distribution
}

\author{
Cecilia B. M. H. Chirenti \\ Instituto de Física, Universidade de São Paulo \\ C.P.66.318, CEP 05315-970, São Paulo, SP, Brazil \\ (Received on 7 October, 2005)
}

\begin{abstract}
We have developed an algorithm that numericaly computes the dimension of an extremely inhomogeneous matter distribution, given by a discrete hierarchical metric. With our results it is possible to analise how the dimension of the matter density tends to $d=3$, as we consider larger samples.
\end{abstract}

Most authors believe that the large scale distribution of matter should be a constant, as predicted by Einstein's cosmological principle, but there is also a belief that such an idea should be further checked against observation [1]. Some authors argued quite convincingly that the large scale distribution of matter should be fractal, quite the oposite to that expected by the cosmological principle. Inhomogeneous cosmological models have been searched by many authors [2] [3] [4] [5] [6] [7]. The fact that detailed maps show quite strong inhomogeneities has been a motivation for long controversies [8] [9].

In a previous work [10], we have presented a study on a toy model given by the hierarchical metric

$$
d S^{2}=d t^{2}+g_{11} d x^{2}+g_{22} d y^{2}+g_{33} d z^{2},
$$

where the metric is defined on all integers, depending on their decomposition in terms of powers of 2 as

$$
\begin{array}{ll}
g_{11}(x)=a(t)^{2 k}, & \text { with } \quad x=2^{k+1} n+2^{k}-1 \\
g_{22}(y)=a(t)^{2 \ell}, & \text { with } \quad y=2^{\ell+1} n+2^{\ell}-1 \\
g_{33}(z)=a(t)^{2 m}, & \text { with } z=2^{m+1} n+2^{m}-1,
\end{array}
$$

where $k, \ell, m$ and $n$ are integers. We obtained the following expression for the matter density,

$$
\begin{aligned}
T_{00} \equiv \rho= & \frac{1}{8 \pi G} \frac{\dot{a}^{2}}{a^{2}}(k \ell+\ell m+m k) \\
& \equiv \rho_{0}(t)(k \ell+\ell m+m k),
\end{aligned}
$$

by means of the Einstein equations. Computing the Christoffel symbols and subsequently the curvature tensor for this metric requires some care, since we are not dealing with derivatives of functions, but differences of functions defined on a discrete space.

We speculated whether such a matter distribution could be described by a fractal. As it turned out, from our preliminary analisis (see [10]), the dimension of the matter density tends slowly to $d=3$.

Considering the relation

$$
\lim _{r \rightarrow \infty} \frac{N(r)}{r^{d}}=K, \quad \text { with } N(r)=\sum_{0<x, y, z<r} \frac{\rho}{\rho_{0}},
$$

where $K$ is some constant, and the constant $d$ is the fractal dimension of the matter density [11], it is easy to see that it implies

$$
\ln N(r)=\ln K+d \ln r
$$

for large $r$. We have numerically computed $N(r)$ and the plot $\ln N(r) \times \ln r$, in figure (1), showing that equation (5), although valid at very large $r$ ), is a good approximation for the behavior of the data.

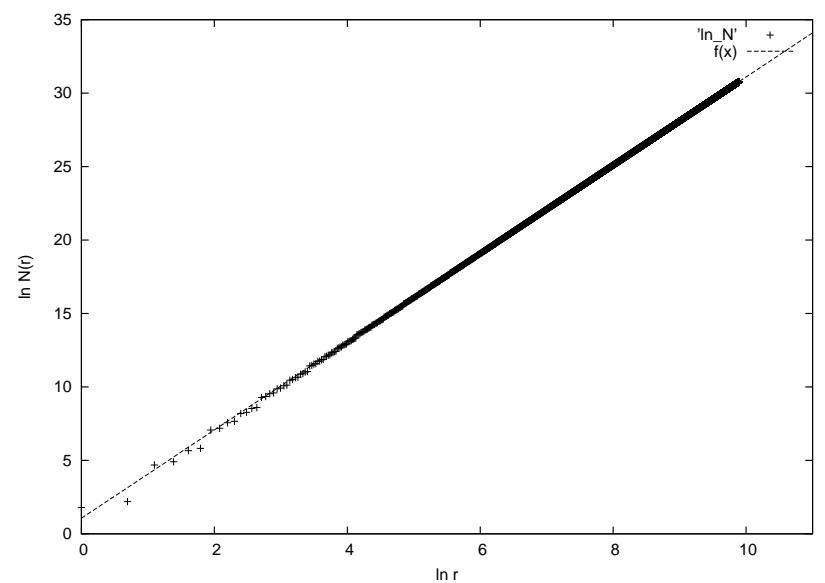

FIG. 1: In this graphic we have $\ln N(r) \times \ln r$. The equation of the straight line $f(x)=a x+b$ has $a=3.00374 \pm 0.000025$, $b=1.06288 \pm 0.000225$.

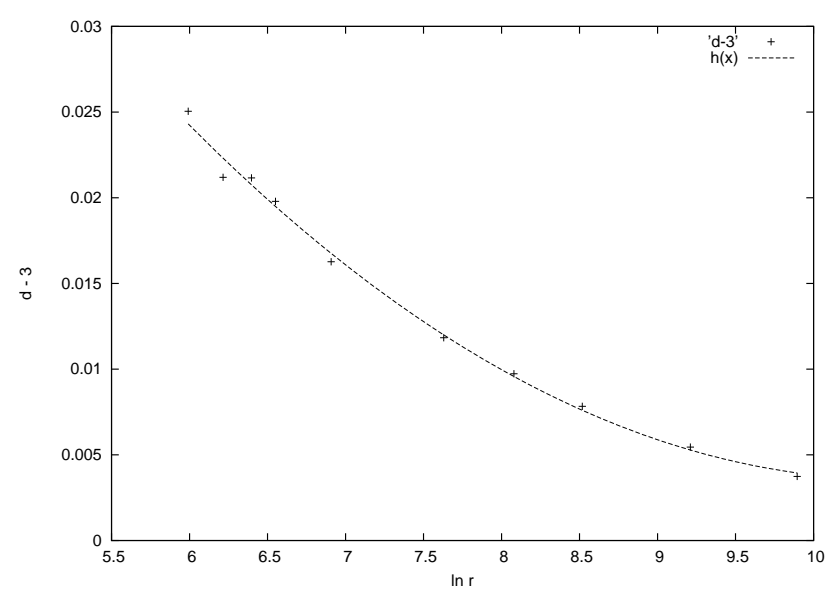

FIG. 2: This graphic shows $(d-3) \times \ln r$, obtained for $r$ values up to 20.000 . The curve is a parabola fitted to the points by Gnuplot, with equation $h(x)=a x^{2}+b x+c: a=0.00102 \pm 0.00014$, $b=-0.02134 \pm 0.00220, c=0.11576 \pm 0.008441$. 
Numerical fits for these points, taking increasingly larger samples, have given results for $d$ that approach $d=3$, as shown in figure (2).

As a conclusion, we can say, now, that the dimension of the matter density that generates metric (1) tends to $d=3$ as shown in figure (2).
Acknowledgements: This work has been supported by Fundação de Amparo à Pesquisa do Estado de São Paulo (FAPESP), Brazil.
[1] P. H. Coleman and L. Pietronero Phys. Rep. 231, 311 (1992).

[2] G. Lemaître Ann. Soc. Sci. Bruxelles A53, 51 (1933); R. C. Tolman Proc. Nat. Acad. Sci. USA 20, 169 (1934); H. Bondi Mon. Not. Roy. Astr. Soc. 107, 410 (1947).

[3] A. Krasiński Inhomogeneous Cosmological Models Cambridge University Press (1997).

[4] M. B. Ribeiro Astrophysical Journal 388, 1 (1992); 395, 29 (1992); 415, 469 (1993).

[5] E. Abdalla, N. Afshordi, K. Khodjasteh, and R. Mohayaee Astr. and Astro. 345, 22 (1999), astro-ph/9803187.

[6] Elcio Abdalla and M. Reza Rahimi Tabar Phys. Lett. B440, 339 (1998), hep-th/9803161.
[7] E. Abdalla and R. Mohayaee Phys. Rev. D59, 084014 (1999), astro-ph/ 9810146; Braz. J. Phys. 31, 42 (2001), astro$\mathrm{ph} / 9811119$.

[8] M. B. Ribeiro, Gen. Rel. Grav. 33, 1699 (2001), astro$\mathrm{ph} / 0104181$.

[9] E. Abdalla, M. B. Ribeiro, and R. Mohayaee Fractals 9, 451 (2001), astro-ph/9910003.

[10] E. Abdalla, C. B. M. H. Chirenti, Phys. A 337, 117 (2004) .

[11] B. B. Mandelbrot, The Fractal Geometry of Nature, New York, (1983). 